\title{
Una riflessione sulla formazione in Architettura a Palermo
}

\author{
Andrea Sciascia
}

Mettere a fuoco l'odierna formazione in Architettura a Palermo, invita a tratteggiare un quadro composto da due differenti prospettive: la prima orientata a individuare alcune peculiarità sviluppatesi in circa duecento anni di storia; la seconda limitata a un campo cronologicamente molto più ristretto in grado di porre in risalto gli ultimi decenni. In realtà, la raffigurazione è costituita da un'unica grande immagine, quella del periodo più recente, posta sopra una predella con alcune figure, ognuna delle quali coglie, dai circa due secoli di storia, alcune peculiarità.

Tale figura, nel suo complesso, è il rilievo e contemporaneamente il progetto della $\mathrm{Fa}$ coltà di Architettura la cui eredità è confluita, nel Dipartimento di Architettura ${ }^{\mathrm{I}}$ e, per quanto attiene l'attività didattica, nel corso quinquennale a ciclo unico in Architettura, in una struttura di Ateneo che, dopo la legge 240 del 2010, non prevede più le Facoltà ma i Dipartimenti e le Scuole².

Dopo tale precisazione, la tela della predella è riempita da rapidissimi schizzi, le cui linee sono rese più nitide dai rimandi bibliografici che costituiscono le tracce da sviluppare in altre occasioni di approfondimento. Da una sintesi estrema si estraggono, un po' ex abrupto, alcune costanti individuando, ad esempio, nell'apporto «straniero» di Léon Dufourny, presente in Sicilia alla fine del XVIII secolo, un aspetto saliente dell'insegnamento di Giuseppe Venanzio Marvuglia, capostipite dei docenti di architettura ${ }^{3}$ alla Regia Accademia degli Studi ${ }^{4}$. All'architetto francese, autore del Gymnasium dell'Orto Botanico di Palermo, cuore della cultura positivista della città, si deve un nuovo concetto di architettura fondata sull'evidenza oggettiva dei rinvenimenti archeologici piuttosto che sul dictatum della trattatistica storica, traendo, inoltre, dallo studio e dalle interpretazioni delle vestigia architettoniche disseppellite, nuovi e originali temi formali utilizzabili all'interno del progetto di architetturas.

La lezione di Dufourny, sulla scia dei tanti architetti europei attenti conoscitori dell'archeologia siciliana, trasforma il territorio dell'isola nel primo testo da cui i docenti e gli allievi apprenderanno.

Dopo l'Unità d'Italia le qualità della formazione palermitana, bilanciata tra aspetti scientifici e umanistici, rischiano di essere compromesse dall'applicazione della legge Casati ${ }^{6}$ tesa a favorire soprattutto il progresso tecnologico e la formazione dei Politecnici. Tale fase che vede la nascita delle Scuole di Applicazioni per Architetti e Ingegneri termina, dopo la «riforma Gentile», con la fondazione nel I935 della Facoltà di Ingegneria. Come filiazione diretta di questa, alla fine della seconda guerra mondiale - conclusasi in Sicilia nel 1943 con lo sbarco degli americani -, è istituita nel I 944 la Facoltà di Architettura ${ }^{7}$. Protagonisti del momento di avvio sono i discepoli e gli epigoni di Ernesto Basile, fra i quali si ricordano: Salvatore Benfratello, Salvatore Caronia Roberti, primo preside della Facoltà, e Edoardo Caracciolo. In seguito

nel decennio degli anni Sessanta tra i cattedratici ci sono presenze interessanti: nella Storia Guido Di Stefano prima e Renato Bonelli dopo, nella Composizione Gino Levi Montalcini prima, poi Luigi Vagnetti ed una fugace presenza di Giulio Roisecco. Completavano il quadro, docenti a contratto annuale, un gruppo di architetti pa- 


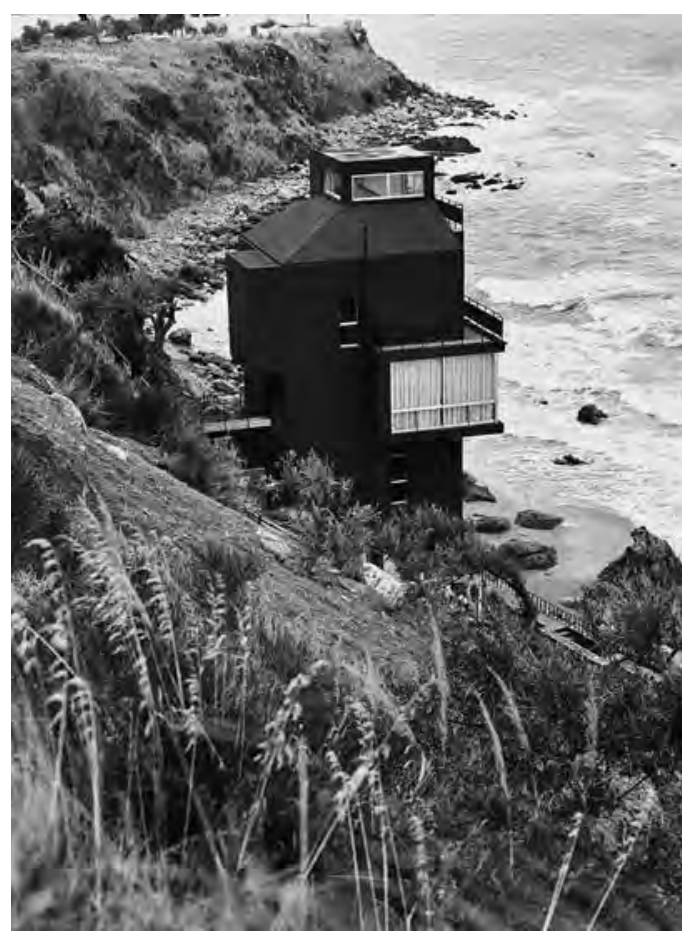

I. Pasquale Culotta, Giuseppe Leone, casa Salem, Cefalù, I 973 .

lermitani professionisti, autori delle realizzazioni che segnarono in Sicilia il passaggio dall'esperienza Liberty, al Novecento razionalista del moderno, quali: Giuseppe Spatrisano, Salvatore Cardella, Luigi Epifanio, Giuseppe Vittorio Ugo, Vittorio Ziino, Giuseppe Caronia .

Nella fase di avvio e, in particolare negli anni Cinquanta, si radicano, soprattutto grazie alla attività di Edoardo Caracciolo, alcune ricerche sui cosiddetti centri minori in cui ritorna con forza, seppur in modo diverso, quel rapporto con il territorio precedentemente enucleato. La riflessione sui sistemi urbani più minuti, sulla scia di altre importanti ricerche italiane, costituisce per gli allievi un modo più immediato per apprendere quelle regole che presiedono alla costruzione dell'architettura e della città. Riescono così ad affinare la loro esperienza nell'architettura sia nella fase di lettura, sia in quella di progetto. Una didattica en plain air, interessata a verificare gli esiti della letteratura architettonica nella concretezza dei luoghi, sembra essere, da una disamina a posteriori, l'ambito di incontro con le innovative ricerche - influenzate dalla fenomenologia e dallo strutturalismo - di Vittorio Gregotti, docente a Palermo dal ig68 sino ai primi anni Settanta.
Prima di Gregotti giunge a Palermo Alberto Samonà, nel 1965 , e sarà lui a trasformare in modo radicale la didattica della progettazione architettonica basata, per i primi due decenni, su una relazione "gomito a gomito»?. Tale modus operandi è ribaltato da Samonà mettendo

a punto un rapporto tra il corso e gli studenti mediato, non più attraverso un rapporto personale tra lui, o i suoi assistenti, e gli studenti, ma attraverso una serie di elementi scritti che erano soprattutto legati alla descrizione ed alla decostruzione di architetture significative $^{10}$.

Con Samonà si diffonde una conoscenza approfondita del Movimento Moderno, e l'approccio al town design.

Dalla allora vigente normativa nazionale sulle cattedre universitarie e da una serie di chiamate a contratto, si apre una stagione nuova a Palermo dove i professori «esterni» ${ }^{\mathrm{II}}$, in prevalenza provenienti da Milano e da Roma, trovano spazio nel corpo docente, sino alla metà degli anni Sessanta, in maggioranza autoctono. Questa fase trova il suo acme nel I973 con il Programma-quadro per l'ordinamento della Facoltà di Architettura proposto da Gino Pollini, Vittorio Gregotti, Gino Lo Giudice e Alberto Samonà.

Un Piano [...] sostanziato dal riconoscimento chiaro e senza equivoci della centralità nella Scuola della pratica del progetto di architettura, costruita su due ambiti di esperienza: il territorio siciliano e quello dell'architettura universale ${ }^{12}$.

La centralità del progetto di architettura costituisce, a distanza di più di quarant'anni dalla sua formulazione, la rotta che ha resistito alle riforme universitarie successive. E fra gli anni Settanta e Novanta, Palermo vive la sua stagione più felice dove, al rinnovato corpo docente si sommano occasioni di confronto con alcuni dei più autorevoli progettisti stranieri ${ }^{13}$ e dove $\mathrm{i}$ «giovani» assistenti palermitani dei docenti «esterni» raggiungono piena maturità. Con la guida di Pasquale Culotta, nei primi anni Settanta assistente di Gino Pollini e preside dal 1989 al 1996, Palermo avrà una ribalta nazionale e internazionale, periodo nel quale gli esiti della produzione didattica e di ricerca trovano spazio alla Triennale di Milano ${ }^{\mathrm{I} 4}$ e alla Biennale di Venezia ${ }^{15}$.

La qualità di questa formazione ha un riscontro in opere costruite nel territorio sici- 


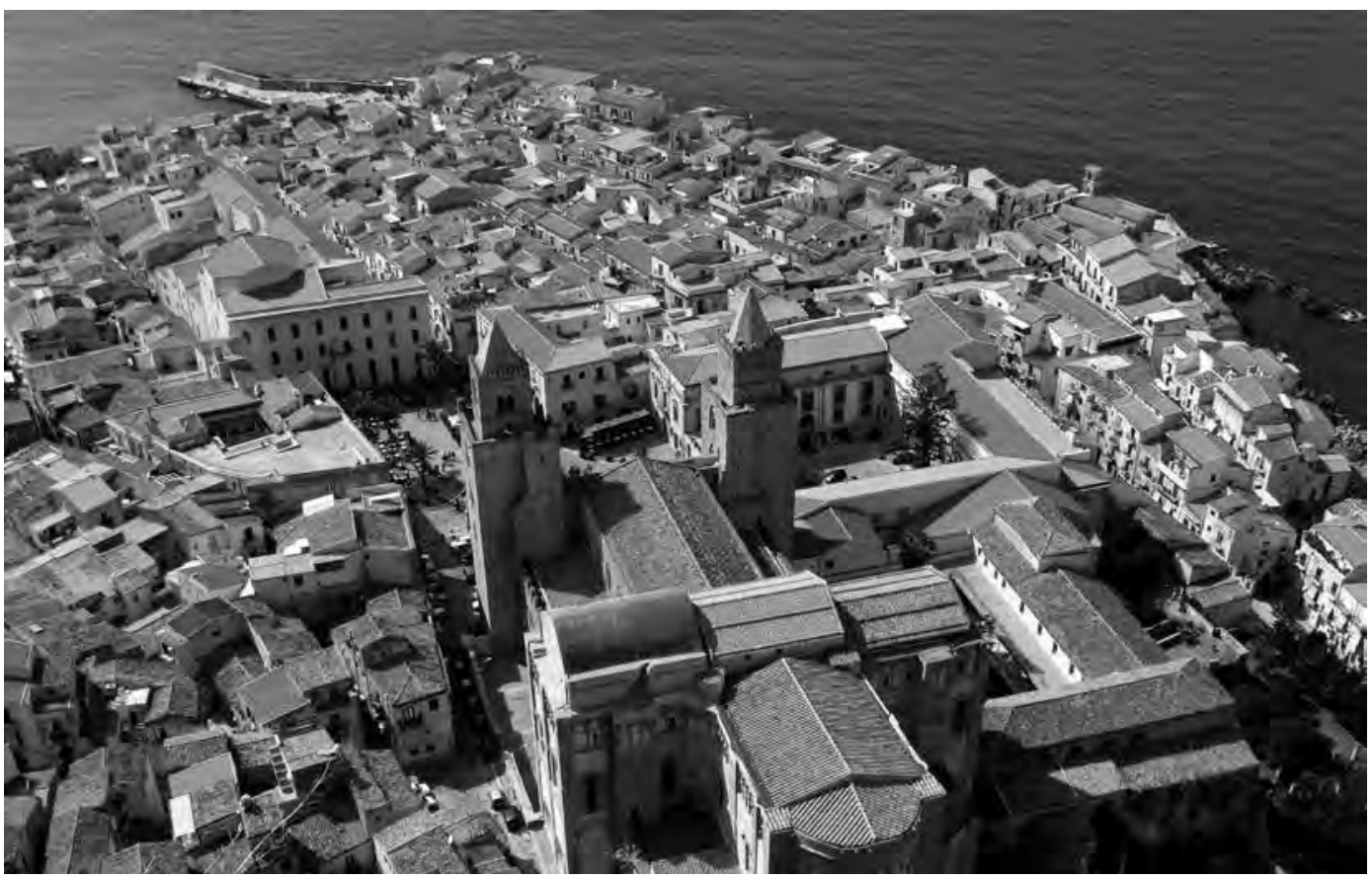

2. Pasquale Culotta, Giuseppe Leone, municipio di Cefalù nel tessuto urbano 198 I-1994. Foto Pubblico Dominio.

liano, in modo molto più sfumato a Palermo, raggiungendo un notevole rilievo pubblicistico. Saranno Amedeo Belluzzi e Claudia Conforti a scrivere come «l'impronta comune della giovane architettura siciliana ha la sua matrice nella scuola di Palermo, guidata da Culotta e Leone, che costituisce forse la novità disciplinare più significativa degli anni Ottanta» ${ }^{16}$.

Il successo della Facoltà è quasi esclusivamente un riscontro extra moenia, cioè al di fuori di Palermo, città che dimostra dalla fine degli anni Ottanta una sostanziale indifferen$\mathrm{za}$ alle ricerche e alla didattica, in alcuni casi inseparabili, dei docenti architetti ${ }^{17}$.

Oggi che si registra una lieve inversione di tendenza nel rapporto tra città e Dipartimento di Architettura, senza infingimenti, si dirada quell'apporto salutare e indispensabile dei docenti progettisti, impegnati a tutto tondo nella didattica, nella ricerca e nella professione. L'osmosi assoluta e vivifica fra questi tre aspetti - didattica, ricerca e professione - sembra ridursi in molte giovani leve della docenza e, conseguentemente, si rischia di rendere più labile il contributo dell'insegnamento. Tale fenomeno, insieme a Palermo, riguarda una parte consistente dei corsi di laurea in Architettura d'Italia e merita una riflessione di ordine complessivo.
Pur con queste ultime note non del tutto positive, le peculiarità di Palermo, poste in evidenza, continuano a trovare un ottimo riscontro nel numero delle iscrizioni e, nel rapporto tra formazione e mondo del lavoro. Quest'ultimo sottolineato da una costante e proficua collaborazione con l'Ordine professionale.

Inoltre le molte relazioni dei docenti con sedi estere consentono, in questo caso, agli allievi di tutti i corsi di laurea grandi possibilità di scelta per il periodo Erasmus ${ }^{18} \mathrm{e}$, conseguentemente, al Dipartimento di Architettura una consistente proiezione internazionale confermata da importanti collaborazioni nella ricerca. Proprio l'attività Erasmus, potenziata con l'Erasmus plus, che consente, di fatto, allo studente di trascorrere quasi metà del proprio percorso formativo all'estero - due anni su cinque - sollecita un altro tipo di considerazioni. Si ritiene, infatti, che alla positiva facilità con cui gli allievi si spostano in quasi tutti $i$ Paesi d'Europa dovrebbe accompagnarsi, con ancora maggiore semplicità, la possibilità di sostenere anche un semestre o un solo esame in un'altra università italiana. Si immagina un sistema che superi le lungaggini burocratiche dei cosiddetti trasferimenti ma che rientri in una procedura spedita; scambi forse attuabili attraverso delle convenzioni fra le varie sedi. 
L'effetto benefico, al quale si dovrebbe accompagnare una mobilità dei professori, arricchirebbe la conoscenza, in generale, e la cultura del progetto, in particolare, permettendo alle singole strutture didattiche dei corsi di laurea una continua verifica diffusa. La struttura del corso di laurea in Architettura di Palermo prevede pochissimi corsi integrati, preferendo un coordinamento e quindi una interdisciplinarietà cercata volontariamente dai docenti e non imposta da un manifesto degli studi. Tale modo dà responsabilità ai professori e trasforma ogni anno del corso di laurea, tendenzialmente, in una occasione di sperimentazione didattica. Ad esempio, al primo anno, vi è stata una convergenza dei laboratori di Progettazione architettonica su un unico programma tema, luogo e bibliografia in comune - e, attorno a questo nucleo, si è sviluppata una intesa con gli altri colleghi e quindi con i contenuti di tutti gli insegnamenti. Tale circostanza ha permesso di sviluppare da Palermo, dall'anno accademico $2015-2016$, un coordinamento nazionale dei laboratori di prima annualità ${ }^{19}$; attività che andrà avanti almeno per il prossimo triennio e che ha già prodotto una occasione di confronto nazionale ${ }^{2 \circ}$. Si pensa che le opportunità di discussione vadano moltiplicate per comprendere sino in fondo quale contributo ogni sede saprà dare, in uno scenario sempre più mutevole, per migliorare la formazione architettonica in Italia. Pur nei limiti dell'ultima riforma universitaria, è concreta la possibilità di apportare ponderati interventi di «manutenzione ordinaria» ai corsi di laurea, in un'azione che può giovarsi del punto di vista dei dipartimenti, laddove su questi convergano le responsabilità della didattica, della ricerca e della terza missione. Quest'ultima attività, che ormai rientra nei parametri soggetti a valutazione, implica una relazione diretta fra dipartimenti e territorio, consente ai primi un reale trasferimento delle conoscenze acquisite per contribuire allo sviluppo sociale, culturale ed economico della società. Mostrata la specificità di questo compito, la terza missione sembra essere soltanto un modo nuovo per chiamare un aspetto strutturale degli studi di architettura; il punto di saldatura di quella operosità e necessità di rivolgersi all'esterno. L'esplicitazione di un rapporto biunivoco che implica un abbeverarsi alla fonte - territorio, città, architettura - restituendo quanto appreso in termini di conoscenza e progetto.
Note

Il Dipartimento di Architettura (DARCH), costituitosi nel 2010 in seguito alla legge Gelmini (240/2010) e alla confluenza dei Dipartimenti Città e territorio, Design, Progetto e costruzione edilizia, Rappresentazione, Storia e progetto nell'architettura, svolge gran parte delle attività della stessa Facoltà di Architettura, ereditandone la lunga tradizione di studi. L'offerta formativa del Dipartimento di Architettura, per l'a.a. 20I6-2017, è costituita dai seguenti cinque corsi di studi: corso di laurea triennale in Disegno industriale, classe $\mathrm{L}_{4}$, coordinatore professore Dario Russo; corso di laurea triennale in Scienze della pianificazione territoriale, urbanistica, paesaggistica e ambientale, classe L2I; corso di laurea magistrale in Pianificazione territoriale, urbanistica e ambientale, classe $\mathrm{LM}_{4} 8$, entrambi coordinati dal professore Francesco Lo Piccolo; corso di laurea magistrale a ciclo unico in Architettura, classe $\mathrm{LM}_{4}$, coordinatore professore Vincenzo Melluso; corso di laurea magistrale a ciclo unico in Ingegneria edile-architettura, classe $\mathrm{LM}_{4}$, coordinatore professore Giuseppe Pellitteri. Le ricerche e le competenze del DARCH investono l'intero ambito delle analisi, delle indagini e delle trasformazioni dell'ambiente fisico, e vanno: dalla progettazione architettonica, urbana e paesaggistica, alla pianificazione urbana e territoriale; dal processo progettuale finalizzato alla costruzione, all'indagine storica, al rilevamento dei beni architettonici rivolti alla diagnostica, al restauro e alla loro valorizzazione.

Il Dipartimento di Architettura conferisce i propri corsi di laurea alla Scuola Politecnica, presieduta dal professore Maurizio Carta.

3 Furono successori di Marvuglia nella cattedra di Architettura civile, il figlio Alessandro Emmanuele (dal I 8 I 4 al i 8 I 5), Cristoforo Cavallaro (dal i 8 is al i 8 I 7), Antonio Gentile (dal I 8 I 8 al I 834), Giuseppe Caldara (dal I 834 al i 837), Carlo Giachery (dal I 837 al i 865). Con l'attivazione della Scuola d'Applicazione per gli Ingegneri e Architetti nel i 866, fu istituita la cattedra di Esercizi di composizione architettonica affidata a Giovan Battista Filippo Basile, già professore ordinario di Architettura decorativa e composizione.

4 La Regia Accademia degli Studi fu fondata nel I779 nell'ambito del Piano della riforma dei pubblici Studi della Capitale e del Regno. Si componeva di quattro facoltà: Teologica, Medica, Giuridica e Filosofica. All'interno di quest'ultima fu inserita la cattedra di Geometria pratica e Architettura civile e idraulica, affidata a Giuseppe Venanzio Marvuglia. Il passaggio da Accademia degli Studi a Università fu sancito con regio decreto del 12 gennaio i 806.

5 G. Di Benedetto, La scuola di architettura di Palermo, I779-I865, in C. Ajroldi (a cura di), Per una storia della Facoltà di Architettura di Palermo, Officina, Roma 2007, p. 62.

6 Legge sulla Pubblica Istruzione n. 3725, promulgata a Torino il I 3 novembre I 859 .

La Facoltà di Architettura di Palermo è rimasta l'unica della Sicilia sino al I996, data di istituzione della 
Facoltà di Architettura dell'Università di Catania, con sede a Siracusa, alla quale si è aggiunta la Facoltà di Ingegneria e Architettura dell'Università Kore di Enna nel 2005.

8 P. Culotta, L'architettura in sessantadue anni nella Sicilia contemporanea, in C. Ajroldi (a cura di), Per una storia della Facoltà di Architettura di Palermo cit., p. 3i. Nello stesso volume si confrontino i testi su: Guido Di Stefano e Renato Bonelli, di Maria Giuffrè, La storia dell'architettura e della città. Maestri, allievi, maestri...: una storia continua, pp. 225-230; su Gino Levi Montalcini, di Anna Maria Fundarò, Il disegno industriale, pp. 277-295.

9 A.M. Fundarò, Il disegno industriale, in C. Ajroldi (a cura di), Per una storia della Facoltà di Architettura di Palermo cit., p. 288.

1o C. Ajroldi, L'insegnamento della composizione a Palermo, Alberto Samonà, 1966-1976, in ivi, p. 2 I I.

${ }^{11}$ Fra i docenti che hanno insegnato Composizione architettonica e urbana a Palermo, per periodi più $o$ meno lunghi, si ricordano: a partire dalla metà degli Sessanta, Carlo Aymonino, Vittorio Gregotti e Alberto Samonà; entro la prima metà degli anni Settanta, Franco Berlanda, Salvatore Bisogni, Tommaso Giura Longo, Sergio Lenci, Carlo Melograni, Pierluigi Nicolin, Gino Pollini, Vieri Quilici, Fabrizio Spirito, Francesco Tentori; tra la fine degli anni Ottanta e la metà degli anni Novanta, Giancarlo Carnevale, Francesco Cellini, Giovanni Di Domenico, Richard Vincent Moore, Manfredi Nicoletti, Angelo Torricelli. Tra i docenti delle altre discipline vanno ricordati: Leonardo Benevolo, Vincenzo Cabianca, Beata Di Gaddo, Carlo Doglio, Roberto Garavini, Enrico Guidoni, Paolo Marconi e Manfredo Tafuri.

I2 P. Culotta, Giovani Architetti Siciliani, "Casabella», s I s, luglio-agosto I985, p. 20.

13 Cfr. F. Alfano, L'insegnamento della progettazione architettonica nella Facoltà di Architettura, 19841994, in C. Ajroldi (a cura di), Per una storia della Facoltà di Architettura di Palermo cit., p. 2 I 5.

I4 Ci si riferisce alla XVII edizione della mostra milanese del 1987, dove Pasquale Culotta è responsabile di un'attività di studio sulla circonvallazione di Palermo. P. Culotta, Nove progetti per l'architettura della circonvallazione di Palermo, in C. De Bevilacqua, A. Ferlenga (redazione del catalogo), Le città immaginate: un viaggio in Italia. Nove progetti per nove città, Electa, Milano 1987, pp. I 82-207.

is Ci si riferisce alla partecipazione alla quinta Mostra Internazionale di Architettura, tenutasi a Venezia dall'8 settembre al 6 ottobre 199I, diretta da Francesco Dal Co, dove Pasquale Culotta espone i lavori degli studenti della Facoltà di Architettura di Palermo all'interno della sezione Venice Prize che, oltre Venezia ospitava, tra quelle italiane, solo la Facoltà di Palermo a confronto con le scuole di ogni parte del mondo. Cfr. G. Guerrera, Pasquale Culotta. Costruire l'avanguardia, catalogo della mostra, Palermo 20I6. Un'altra occasione in cui la Facoltà di Palermo espone alla Biennale di Venezia è l'ottava Mostra Internazionale di Architettura, diretta da Deyan Sudjic, dall'8 settembre al 3 novembre 2002, all'interno della sezione Next. In occasione della mostra del 2002, il preside della Facoltà era Nicola Giuliano Leone, nel ruolo dal 200 al 2007.

I6 A. Belluzzi, C. Conforti, Architettura italiana 19441994, Laterza, Roma-Bari 1994, p. 106.

17 Pasquale Culotta, qualche mese prima della sua prematura scomparsa nel 2006 , aveva scritto: $« \mathrm{~Pa}-$ lermo comunque ha avuto e continua ad avere un ruolo centrale quale campo degli studi d'architettura. Per la Facoltà la città è laboratorio di continue ispezioni didattiche e di approfondimenti scientifici, spesso occasioni di esposizioni pubbliche e di testi scientifici. Tuttavia, di queste attività e del progetto scientifico sugli studi e sulla progettazione urbana che la Facoltà vanta, sono irrilevanti i riflessi sulle decisioni politiche, burocratiche e culturali decisive per l'architettura del territorio della città», P. Culotta, L'architettura in sessantadue anni nella Sicilia contemporanea cit., p. 33.

I8 In merito all'Erasmus è molto elevato il flusso in uscita (outgoing), mentre è ancora ridotto quello in entrata (incoming).

19 Il coordinamento nazionale Incipit_lab ha proposto un confronto tra i laboratori di Progettazione architettonica di prima annualità e ha visto l'adesione di quasi tutte le Scuole di Architettura italiane: I. Boniello, A. Chirotti, M. Landsberger, A. Lorenzi, T. Monestiroli, S. Rolla, L.M. Spinelli (Politecnico di Milano); F. Cacciatore (Istituto Universitario di Architettura di Venezia); E. Narne (Università di Padova); C. Ravagnati (Politecnico di Torino); C. Gandolfi (Università di Parma); D. Durante, A. Stella, A. Rinaldi (Università di Ferrara); P. Bonvini (Università di Ancona); S. Ferrini (Università di Chieti-Pescara); D. Nencini (Sapienza Università di Roma); A. Falzetti (Università di Roma Due); L. Franciosini (Università di Roma Tre); R. Capozzi, A. Picone, P. Scala, F. Visconti (Università di Napoli Federico II); A. Russo (Università di Reggio Calabria); B. Messina (Università di Catania); A. Sciascia (coordinatore Incipit_lab), G. Di Benedetto, G. Marsala, N. Margagliotta (Università di Palermo). Tale raffronto scaturisce dal desiderio e dalla necessità di verificare e di mettere a sistema, su scala nazionale, le modalità di avvio e di «inizio» (incipit) della prima esperienza di progettazione architettonica nell'ambito della didattica del laboratorio.

${ }^{20}$ Nel luglio 2016 si è svolto, presso il Dipartimento di Architettura di Palermo, il primo convegno nazionale Incipit Lab. Geografie della Didattica. 